\title{
Grip Strength as a Screening Index for Severe Degenerative Cervical Myelopathy in Primary Care: Development of Cutoff Values Using Receiver Operating Curve Analysis
}

\author{
Hiroshi Kobayashi (DD \\ Koji Otani (iD \\ Takuya Nikaido (D) \\ Kazuyuki Watanabe $\mathbb{D}$ \\ Kinshi Kato (D) \\ Junichi Handa \\ Shoji Yabuki (D) \\ Shin-Ichi Konno
}

Department of Orthopaedic Surgery, Fukushima Medical University School of Medicine, Fukushima, Japan
Correspondence: Hiroshi Kobayashi I-Hikarigaoka, Fukushima, 960-I295, Japan

Tel +8I24-547-1276

Fax +8I 24-548-5505

Email hiroshik@fmu.ac.jp
Purpose: Early diagnosis of degenerative cervical spondylosis (DCM) is desirable because late treatment can lead to irreversible sequelae. No screening method has yet been established. Grip strength is commonly used in primary care settings to evaluate disease activity and diagnose sarcopenia. This single-center, cross-sectional study aimed to determine the diagnostic accuracy of grip strength for cervical myelopathy (DCM) and cutoff values for primary care DCM screening using area under the curve (AUC) and sensitivity values.

Patients and Methods: The DCM group comprised 249 consecutive participants (165 males, 84 females; mean age, 65.1 years) with DCM who had undergone surgery at the affiliated hospital. The control group comprised 735 (280 males, 455 females; mean age, 65.8 years) participants undertaking a local government health checkup. Stratifying by age and sex, receiver operating characteristic (ROC) analyses were constructed for each group using minimum grip strength values for both hands. Based on ROC analysis, cut-off values were established so that the screening sensitivity would be $90 \%$ for either sex or age group, respectively.

Results: According to age group and sex (males/females [M/F]), AUC values for a diagnosis of DCM in M/F were as follows: 40-59 years, $0.92 / 0.87 ; 60-69$ years, $0.94 / 0.89 ; 70-79$ years, 0.89/0.91; and 80-89 years, 0.97/0.97. Calculated M/F cutoff values were 41/24.5, 27/ $16,27 / 15$, and $20 / 10 \mathrm{~kg}$, which were similar to cutoff scores for sarcopenia in $\mathrm{M} / \mathrm{F}$ patients aged 60-69 and 70-79 years. M/F sensitivities in each age groups were $0.94 / 0.91,0.92 / 0.90$, $0.95 / 0.96$, and $0.92 / 0.93$. $\mathrm{M} / \mathrm{F}$ specificities were $0.62 / 0.59,0.84 / 0.83,0.61 / 0.71$, and $0.83 /$ 0.88 .

Conclusion: Grip strength had moderate-to-high diagnostic accuracy for DCM between participants in the control and DCM groups. We developed easily applicable cutoff values for primary care DCM screening with $\geq 90 \%$ sensitivity. In patients with sarcopenia, DCM should be differentially diagnosed in primary care.

Keywords: degenerative cervical myelopathy, cervical spondylotic myelopathy, ossification of posterior longitudinal ligament, cervical disc herniation, primary care, early detection, sarcopenia

\section{Introduction}

Early detection of degenerative cervical myelopathy (DCM) is becoming increasingly important because of the expected increase in patient numbers within aging populations, with a consequent increased risk of falls resulting in spinal cord injury 
and fracture. ${ }^{1-5}$ Patients with undiagnosed DCM may consult their primary care provider when they first experience symptoms such as numbness or clumsiness in the hands and gait disturbances. Nonetheless, a diagnosis of DCM is often challenging. If DCM could be easily screened in primary care, surgery at an appropriate time point may prevent exacerbation of this disease and improve activities of daily living (ADL), contributing to the extension of healthy life expectancy in aging societies.

Clinical tests and measures to rule out DCM have included the Hoffmann test, the finger escape sign, the Babinski sign, Lhermitte's sign, the 10 -second grip and release test, and a patient-oriented questionnaire. ${ }^{6-13}$ However, screening in primary care settings using these methods does not appear to be widespread.

We focused on grip strength in this study as it has been commonly used to assess DCM in orthopedic clinical practice. It has also been used in internal medicine to evaluate disease activity. ${ }^{14}$ Furthermore, it is increasingly being measured in primary care to evaluate sarcopenia, a condition characterized as a loss of skeletal muscle mass and strength in older adult patients. ${ }^{15}$ This test can be introduced at low cost and can be measured in advance by healthcare professionals other than doctors, to observe changes over time. Nonetheless, the diagnostic accuracy of grip strength in DCM remains unknown.

This study aimed to determine the diagnostic accuracy of grip strength for DCM using receiver operating characteristic (ROC) analyses and aimed to develop cutoff values to screen for DCM that can be easily applied in primary care settings.

\section{Materials and Methods}

\section{Ethical Considerations}

This study was approved by the Ethics Committee of Fukushima Medical University and was conducted in accordance with the Declaration of Helsinki. Informed consent was obtained in the form of opt-out on the website.

\section{Study Design}

This single-center, cross-sectional study used prospectively collected data to validate diagnostic accuracy involving participants in a DCM group. Participants comprised those visiting the authors' affiliated hospital and individuals in a control group who had participated in a local government health check-up program.

\section{Study Participants DCM Group}

Patients with DCM aged 40-89 years who had undergone surgery at the authors' affiliated hospital from May 2005 to April 2017 were consecutively included in the DCM group. Indications for surgery were determined by consensus of at least three board-certified spine surgeons. Patients with a history of cervical spine surgery, cervical trauma, pyogenic spondylitis, cerebrovascular disease, rheumatoid arthritis (RA), destructive spondyloarthropathy, atlantoaxial subluxation, pseudotumor of dens, cervical spondylotic amyotrophy, cerebral palsy, or malformation of the cervical spine were excluded.

\section{Control Group}

The control group comprised individuals aged 40-89 years who had participated in a local government medical check-up program in the towns of Tateiwa, Ina, and Tadami in Fukushima Prefecture, Japan, and who had undergone a cervical spine check-up. The check-up was part of an epidemiological study conducted in 2005, involving local residents with musculoskeletal disorders. ${ }^{16}$ Further, individuals with a history of cerebrovascular disease were excluded. Individuals with possible symptoms of myelopathy, such as hand numbness and clumsy hands, were also excluded from interviews and physical examinations by orthopedic surgeons to rule out individuals with a potential diagnosis of DCM. Specifically, individuals with signs and symptoms that included hand dysfunction, numbness in both hands, pain, or numbness radiating to the upper extremities, and spinal signs such as positivity for Jackson's head compression test, the Spurling test, and Jackson's shoulder depression test were excluded. ${ }^{17,18}$

\section{Grip Strength}

In the DCM group, grip strength measurements were performed the day before or on the day of surgery. In the control group, measurements were taken following an examination to rule out findings suggestive of DCM.

An orthopedic surgeon performed measurements for both groups. The grip strength meter was measured using an EKJ080 (Evernew, Tokyo, Japan). This grip strength meter is an analog type and can measure every $0.5 \mathrm{~kg}$. All assessors were instructed to measure participants in a standing position, except for those who were unable to stand. Bilateral grip strength was measured once, and blinding was not applied to either group. 


\section{Statistical Analyses}

The mean values for grip strength in both hands were compared between the two groups in terms of sex and age using a Wilcoxon rank-sum test, and P-values $<0.05$ were considered statistically significant. ROC analysis was performed for four participant parameters: minimum, maximum, mean of both hands, and left-right differences in grip strength between the two groups stratified according to sex and age group (40-59, 60-69, 70-79, and 80-89 years). The area under the curve (AUC) was calculated. Delimited cutoff values were then set for each group according to age and sex, where sensitivity was $\geq 90 \%$. Finally, sensitivity and specificity values, as well as the positive likelihood ratio ( $\mathrm{LR}+$ ), the negative likelihood ratio (LR-), and diagnostic odds ratios (DORs) of the cutoff values, were calculated. All statistical analyses were performed using $\mathrm{JMP}^{\circledR}$ Pro software program version 15.0.0 (NC, USA).

\section{Results}

In the DCM group, 105 of 368 participants were excluded, including those with a history of cervical spine surgery $(\mathrm{n}=$ $31)$, cervical trauma $(n=9)$, pyogenic spondylitis $(n=2)$, cerebrovascular disease $(\mathrm{n}=11)$, RA $(\mathrm{n}=30)$, destructive spondyloarthropathy $(\mathrm{n}=3)$, atlanto-axial subluxation $(\mathrm{n}$ $=7)$, pseudotumor of dens $(n=2)$, cervical spondylotic amyotrophy $(\mathrm{n}=1)$, cerebral palsy $(\mathrm{n}=8)$, and malformation of the cervical spine $(n=1)$. We also excluded 14 participants with missing grip strength data (Figure 1A). Finally, 249 participants (165 males, 84 females; largest age group, 70 79 years; mean age, 65.1 years) were analyzed (Table 1). The DCM group comprised 161 participants with cervical spondylotic myelopathy, 82 participants with ossification of the posterior longitudinal ligament, and 6 participants with cervical disc herniation.

In the control group, 6 of 994 individuals were excluded because of a history of cerebrovascular disease. We excluded 212 individuals because DCM could not be ruled out based on interviews and physical examinations, and 41 individuals were excluded because of missing grip strength data (Figure 1B). Finally, 735 participants (280 males, 455 females; largest age group, 70-79 years; mean age, 65.8 years) were analyzed (Table 1 ).

The mean grip strength values in the DCM group in terms of sex (males/ females $[\mathrm{M} / \mathrm{F}]$ and age $(40-59,60$ $69,70-79$, and $80-89$ years; \pm SD) were M/F $26.8 \pm 11.2$ / $17.0 \pm 7.3 ; 20.1 \pm 6.4 / 12.5 \pm 5.6 ; 19.3 \pm 7.3 / 10.1 \pm 5.1$; and 12.6 $\pm 6.1 / 6.3 \pm 4.0 \mathrm{~kg}$, respectively (Table 1), whereas the mean grip strength values in the control group were M/F $44.8 \pm 6.6 / 26.8 \pm 6.8 ; 37.2 \pm 7.1 / 21.6 \pm$ $4.5 ; 31.8 \pm 6.8 / 19.1 \pm 4.7$; and $27.9 \pm 5.6 / 16.4 \pm 3.7 \mathrm{~kg}$, respectively. In terms of sex and age, mean grip strength values were significantly lower in individuals in the DCM group than in those in the control group $(\mathrm{P}<0.0001)$. Supplementary Data shows the minimum, maximum, average, and laterality of bilateral grip strength $(\mathrm{kg})$ according to sex and age in the two groups.

\section{ROC Analyses}

The AUC values for minimum, maximum, average, and laterality between the two hands in grip strength are shown in Table 2. Of these, we chose the minimum value between the two hands for our ROC analysis because it showed the highest trend in the AUC.

Figure 2A-H shows the ROC curves determined for minimum grip strength relative to both hands in groups stratified according to age and sex. The age-stratified AUC values for M/F aged 40-59, 60-69, 70-79, and 80-89 years were $0.91 / 0.87,0.94 / 0.91,0.89 / 0.91$, and $0.97 / 0.97$, respectively. A perfect test has an AUC of 1.0, whereas an AUC $>0.9$ indicates high accuracy, an AUC from 0.7 to 0.9 indicates medium accuracy, an AUC from 0.5 to 0.7 indicates low accuracy, and an AUC 0.5 indicates a chance result. ${ }^{19}$

Cutoff values for a diagnosis of DCM in M/F aged 40$59,60-69,70-79$, and 80-89 years were set to $41 / 24.5,27 /$ $16,27 / 15$, and $20 / 10 \mathrm{~kg}$, respectively (sensitivity, $\geq 90 \%$; Table 3). If the measured value was equal to or less than the cutoff value, it was considered to be abnormal. Sensitivities for the cutoff values for $\mathrm{M} / \mathrm{F}$ were $0.94 / 0.91,0.92 / 0.90$, $0.95 / 0.96$, and $0.92 / 0.93$, respectively, and specificities were $0.62 / 0.59,0.84 / 0.83,0.61 / 0.71$, and $0.83 / 0.88$, respectively. LR+ values for M/F were 2.46/2.20, 5.78/5.32, 2.46/ 3.34, and 5.35/7.84, respectively. LR- values for $\mathrm{M} / \mathrm{F}$ were $0.10 / 0.15,0.10 / 0.12,0.08 / 0.05$, and $0.10 / 0.08$, respectively. DORs for $\mathrm{M} / \mathrm{F}$ were $23.6 / 14.2,58.3 / 44.2,32.4 / 64.1$, and 53.2/103.6, respectively (Table 3).

\section{Discussion}

In the present study, we found that when the minimum left and right grip strength was used to diagnose participants with DCM who had undergone surgery, the AUC ranged from 0.87 to 0.97 , indicating moderate to high diagnostic accuracy. ${ }^{19}$ Therefore, we consider that the minimum value of right and left grip strength can be used as a screening tool for DCM. 
A

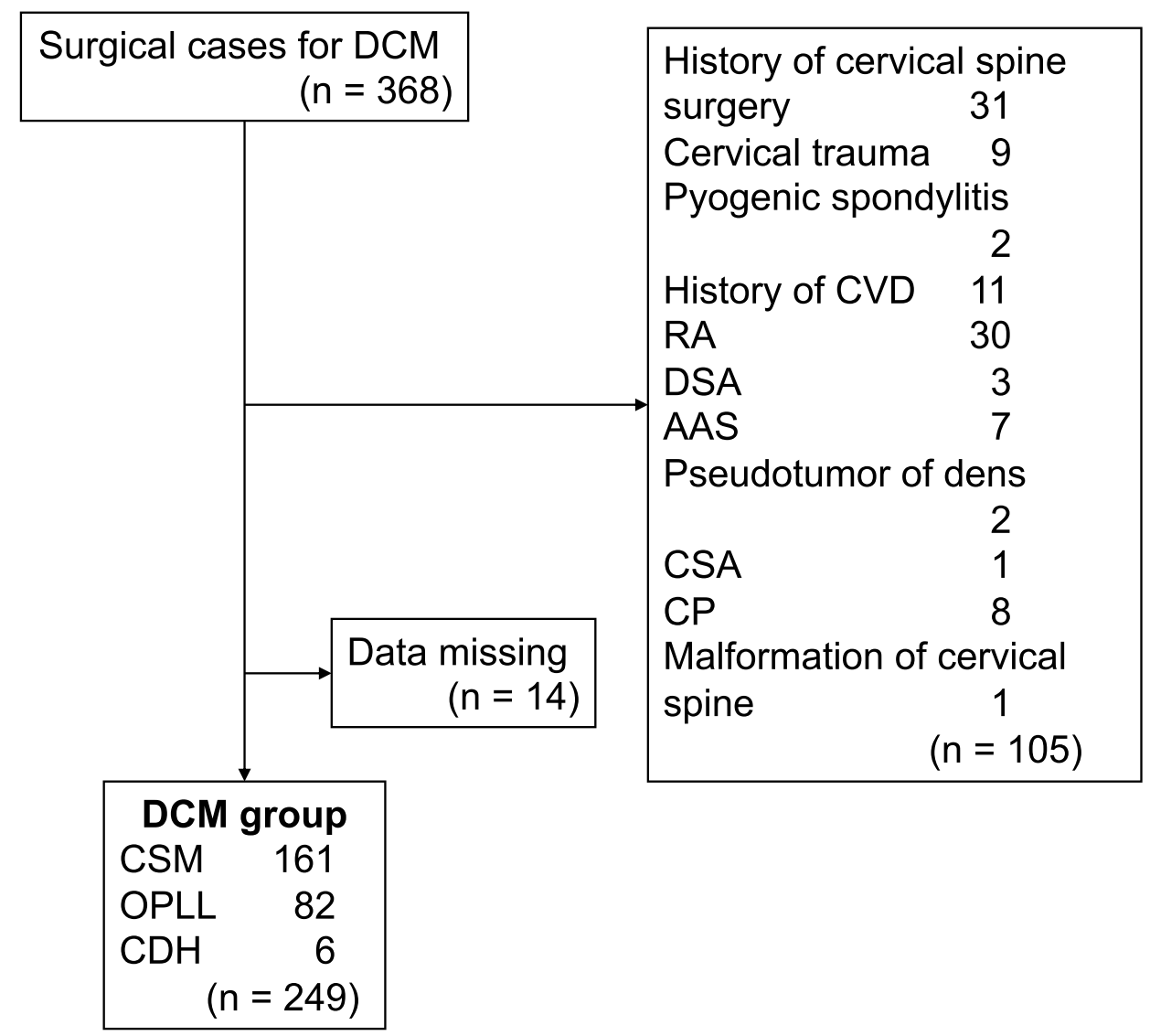

B

Participants of the local government medical checkup

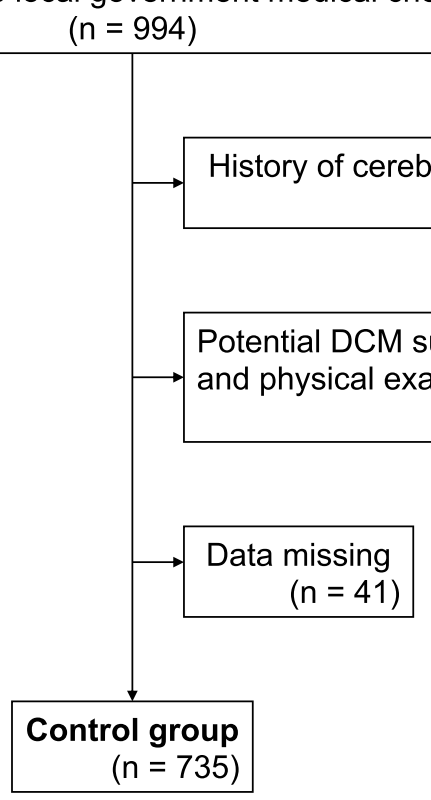

Figure I (A) Flow chart showing DCM group recruitment. (B) Flow chart showing recruitment of the Control group.

Abbreviations: DCM, degenerative cervical myelopathy; AAS, atlantoaxial subluxation; $\mathrm{CDH}$, cervical disc herniation; CP, cerebral palsy; CSA, cervical spondylotic amyotrophy; CSM, cervical spondylotic myelopathy; CVD, cardiovascular disease; DSA, destructive spondyloarthropathy; OPLL, ossification of posterior longitudinal ligament; RA, rheumatoid arthritis. 
Table I Grip Strength by Each Sex and Age Group Between the Two Groups

\begin{tabular}{|c|c|c|c|c|c|c|}
\hline & \multirow[t]{2}{*}{ Age (y) } & \multicolumn{2}{|c|}{$\mathbf{n}$} & \multicolumn{2}{|c|}{ Grip Strength (kg) } & \multirow[t]{2}{*}{$P$ value } \\
\hline & & CTRL & DCM & CTRL & DCM & \\
\hline \multirow[t]{4}{*}{ Male } & $40-59$ & 84 & 63 & $44.8 \pm 6.6$ & $26.8 \pm 11.2$ & $<0.0001$ \\
\hline & $60-69$ & 63 & 48 & $37.2 \pm 7.1$ & $20.1 \pm 6.4$ & $<0.000 \mathrm{I}$ \\
\hline & $70-79$ & 98 & 43 & $31.8 \pm 6.8$ & $19.3 \pm 7.3$ & $<0.0001$ \\
\hline & $80-89$ & 35 & 12 & $27.9 \pm 5.6$ & $12.6 \pm 6.1$ & $<0.0001$ \\
\hline \multirow[t]{4}{*}{ Female } & $40-49$ & 121 & 22 & $26.8 \pm 6.8$ & $17.0 \pm 7.3$ & $<0.0001$ \\
\hline & $60-69$ & 136 & 21 & $21.6 \pm 4.5$ & $12.5 \pm 5.6$ & $<0.0001$ \\
\hline & $70-70$ & 156 & 27 & $19.1 \pm 4.7$ & $\mid 0.1 \pm 5.1$ & $<0.0001$ \\
\hline & $80-80$ & 42 & 15 & $16.4 \pm 3.7$ & $16.4 \pm 3.7$ & $<0.0001$ \\
\hline
\end{tabular}

Abbreviations: CTRL, control; DCM, degenerative cervical myelopathy.

We examined different parameters-minimum, maximum, average, and laterality between right and left grip strength - that would be useful when screening for a diagnosis of DCM. Diagnosis using the minimum right and left-sided grip strength had the highest AUC, that is, the best diagnostic performance for identifying participants with DCM. The reason for this is that DCM generally causes bilateral muscle weakness. However, in the early stages of the disease, there are cases of unilateral weakness; therefore, this method may capture early cases. Conversely, maximum muscle strength and mean are parameters that reflect a decline in grip strength on both right and left sides, and can be considered to indicate a state in which the disease has progressed to some extent. The AUC for laterality in terms of right and left difference was low, and the ability to discriminate between the two groups was limited. Thus, if laterality is detected, cases in which both left and right grip strength have decreased are likely to be missed. Based on these findings, the minimum value of bilateral grip strength can be considered an acceptable screening tool.

This study also identified cutoff values for sex and age to screen DCM with sensitivities of $\geq 0.90$. The cutoff values for both males and females declined sharply in those aged 40-59 years but remained approximately the same in those aged 60-79 years, at $27 \mathrm{~kg}$ for males and $15-16 \mathrm{~kg}$ for females, with a further decline in those aged 80-89 years. Interestingly, the cutoff values in the 60-69and 70-79-year age groups were almost the same as the cutoff values for sarcopenia reported in people of Asian ethnicity (28 kg and $18 \mathrm{~kg}$, respectively). ${ }^{20}$ These findings suggest that if primary care physicians diagnose sarcopenia, DCM should be differentiated, especially in adults aged 60-79 years, regardless of sex.

\section{DCM Screening Methods}

Various clinical tests and measures have been used for DCM screening. The Hoffmann test has been reported to have a sensitivity of $82-94 \%$, whereas sensitivity when blinded to history and clinical findings has been reported to be $28 \%{ }^{6-8}$ The sensitivity of the finger escape sign has been reported to be $55 \% .^{7}$ The Babinski sign has been found to be of variable quality in different reports, and its effectiveness as a screening tool has been questioned. ${ }^{9}$ The sensitivity of Lhermitte's sign has been reported to be

Table 2 AUC

\begin{tabular}{|l|r|r|r|r|r|r|r|r|}
\hline Age Group (y) & \multicolumn{2}{|c|}{ 40-59 } & \multicolumn{2}{c|}{ 60-69 } & \multicolumn{2}{|c|}{ 70-79 } & \multicolumn{2}{|r|}{ 80-89 } \\
\hline Sex & M & F & M & F & M & F & M & F \\
\hline Min & 0.91 & 0.87 & 0.94 & 0.91 & 0.89 & 0.91 & 0.97 & 0.97 \\
Max & 0.91 & 0.81 & 0.91 & 0.88 & 0.89 & 0.9 & 0.98 & 0.93 \\
Ave & 0.93 & 0.84 & 0.94 & 0.89 & 0.89 & 0.91 & 0.98 \\
Lat & 0.61 & 0.69 & 0.66 & 0.6 & 0.65 & 0.52 & 0.49 & 0.96 \\
\hline
\end{tabular}

Abbreviations: AUC, area under the curve; $\mathrm{M}$, male; F, female; Min, minimum grip strength (kg) of the two hands; Max, maximum grip strength (kg) of the two hands; Ave, average grip strength $(\mathrm{kg})$ of the two hands; Lat, laterality of grip strength $(\mathrm{kg})$ between the two hands. 


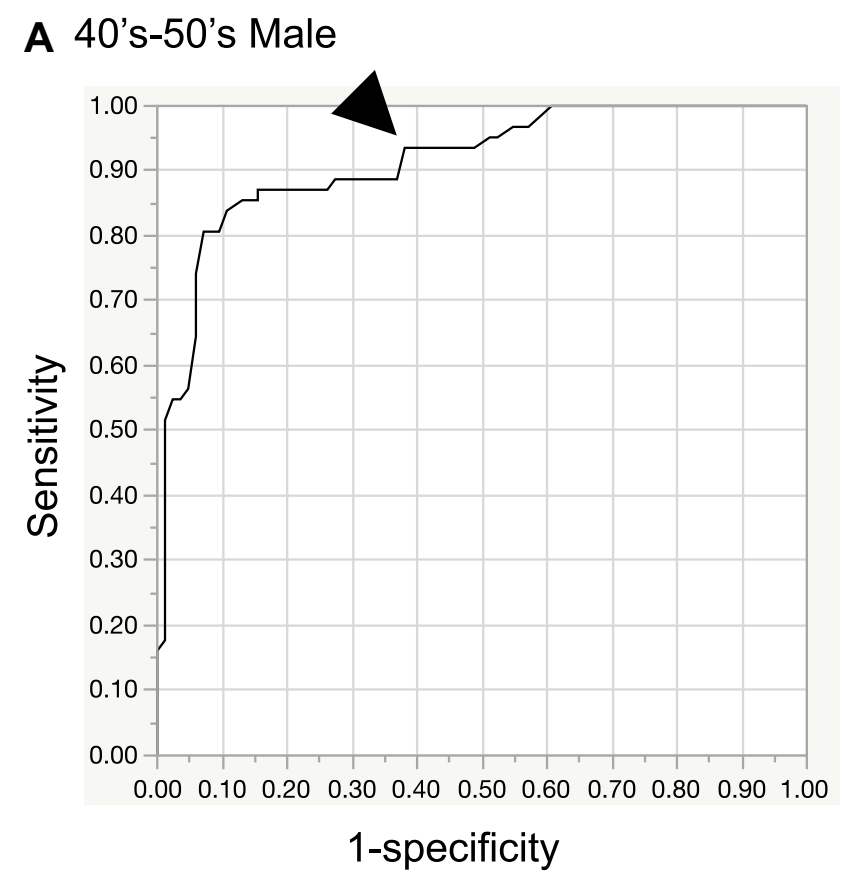

C 60's Male

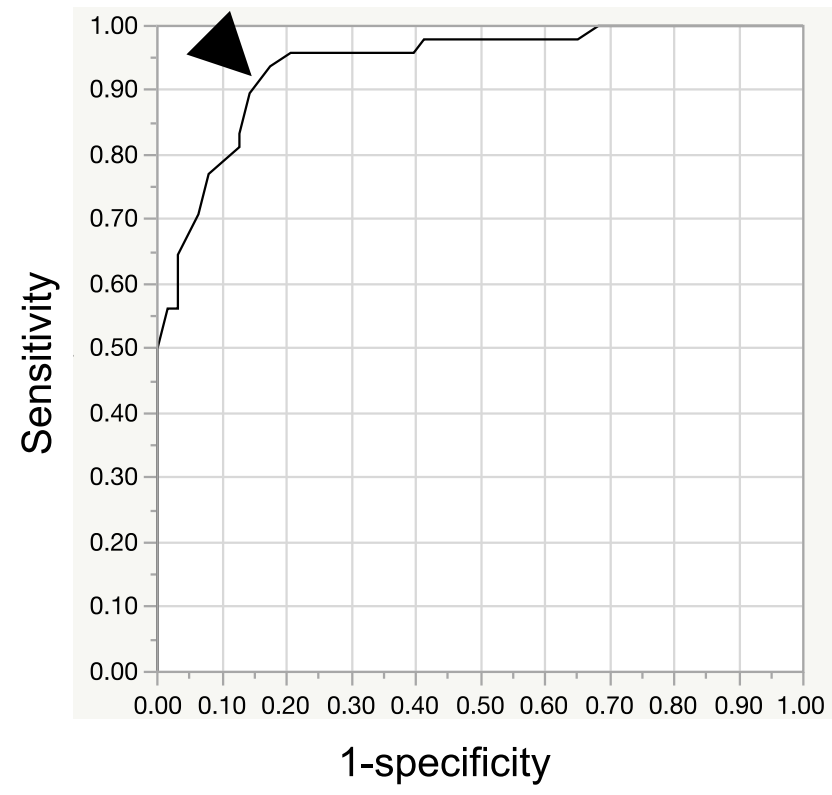

Figure 2 Continue.

remarkably low, ${ }^{10,11}$ and diagnostic accuracy has not been reported for the inverted supinator reflex and clonus test. Therefore, these aforementioned methods were not considered appropriate for DCM screening.

Machino et al reported the diagnostic accuracy of the 10-second grip and release test and cutoff values for DCM diagnosis using ROC analysis. ${ }^{12,21}$ Additionally, Kobayashi et al reported a patient-oriented screening

\section{B 40's-50's Female}
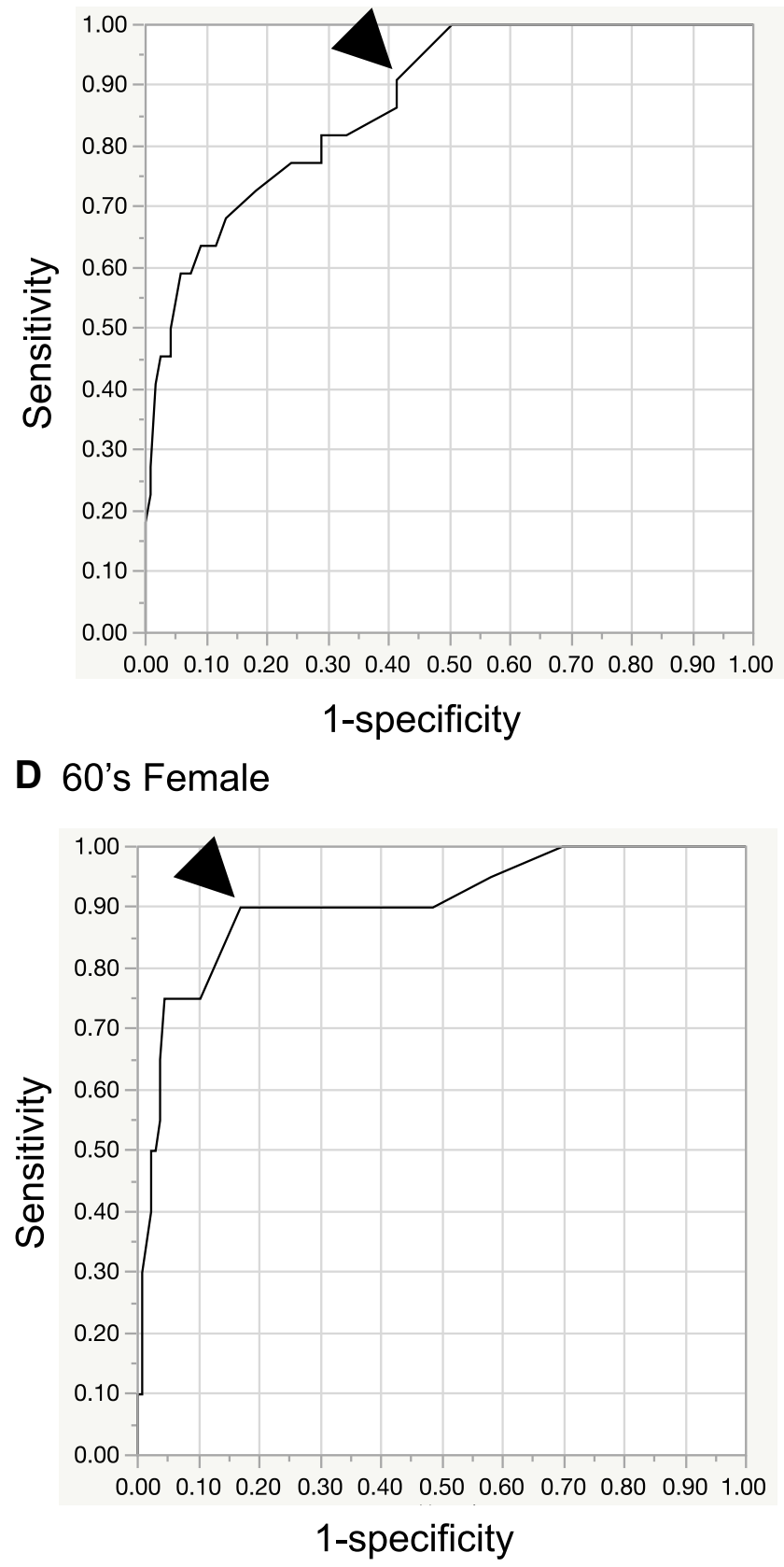

tool for DCM. ${ }^{13}$ They developed their screening tool through extracting questions from the Japanese Orthopaedic Association Cervical Myelopathy Evaluation Questionnaire (JOACMEQ), which is a new version of the Japanese Orthopaedic Association score for cervical disorders, consisting of questionnaires to be used as patient-oriented assessment, and weighting scores for extracted items. ${ }^{22-25}$ This screening tool had 


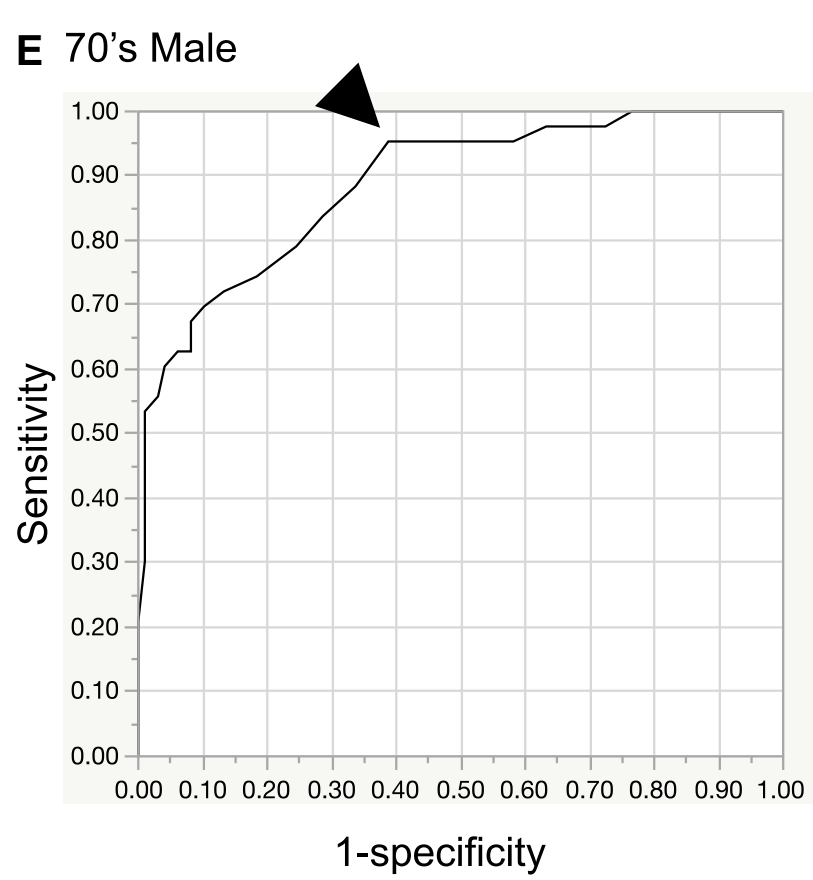

\section{G 80's Male}

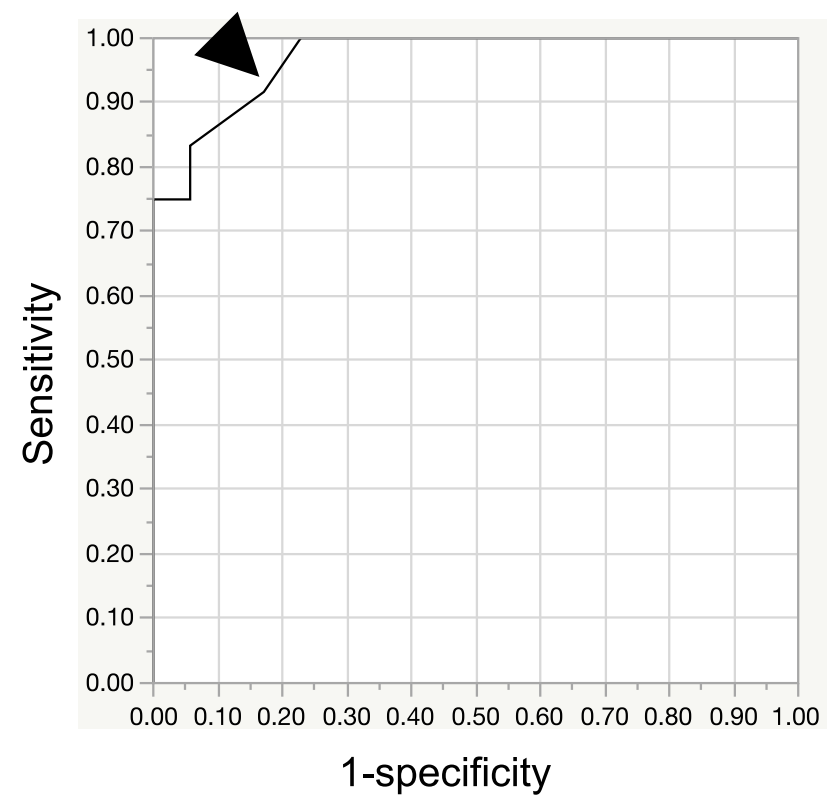

\section{F 70's Female}

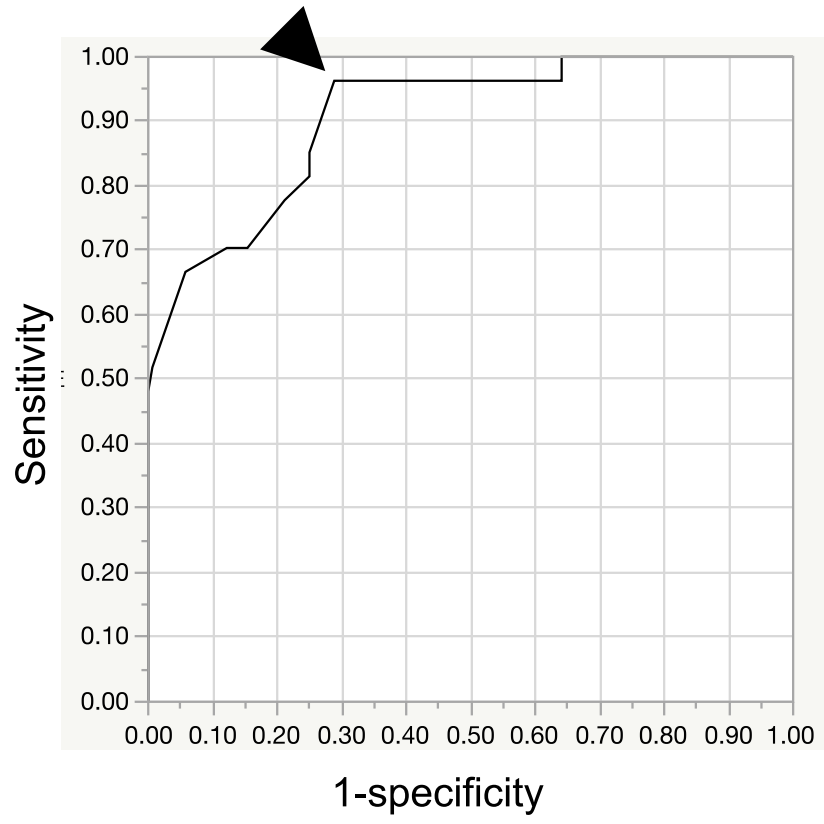

H 80's Female

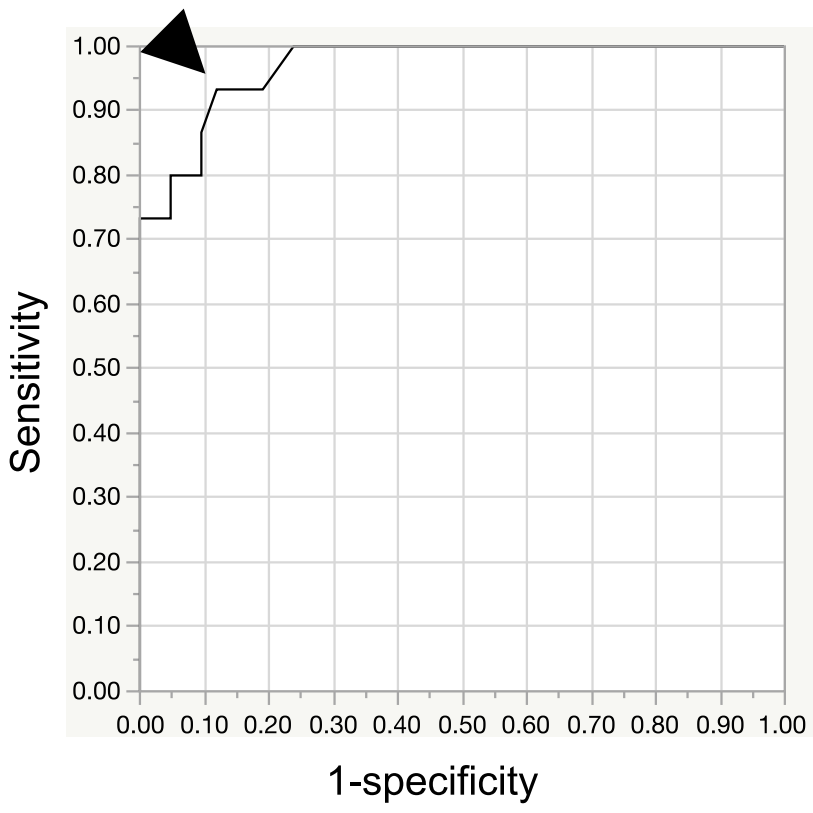

Figure 2 Receiver operating characteristic (ROC) curves by sex and age. The cutoff value (arrowhead) was set at $\geq 90 \%$. (A) 40's-50's male, (B) 40's-50's female, (C) 60's male, (D) 60's female, (E) 70's male, (F) 70's female, (G) 80's male, (H) 80's female.

a sensitivity of $93.5 \%$. These two methods are useful tools for DCM screening; however, these tests are not widely used in primary care. It has been reported that only $5 \%$ of the generalized tests and treatments described in the guidelines are used in primary care. ${ }^{26}$ Therefore, a simpler and more feasible screening method in primary care settings is needed.

\section{Grip Strength}

Grip strength was used as a screening index for DCM. Grip strength is reported to be one of the best indicators of overall limb strength, in addition to being a manageable indicator involving relatively low cost. ${ }^{27}$ Grip strength is commonly used in orthopedic evaluations to assess upper limb muscle weakness due to DCM and to assess 
Table 3 Diagnostic Characteristics of Grip Strength in Each Sex and Age Group

\begin{tabular}{|c|c|c|c|c|c|c|c|c|}
\hline \multirow[t]{2}{*}{ Sex } & \multirow[t]{2}{*}{ Age Group (y) } & Cutoff Value & \multirow[t]{2}{*}{ AUC } & \multirow[t]{2}{*}{ Sensitivity } & \multirow[t]{2}{*}{ Specificity } & \multirow[t]{2}{*}{ LR (+) } & \multirow[t]{2}{*}{ LR (-) } & \multirow[t]{2}{*}{ DOR } \\
\hline & & (kg) & & & & & & \\
\hline \multirow[t]{4}{*}{ Male } & $40-59$ & $4 I$ & 0.92 & 0.94 & 0.62 & 2.46 & 0.1 & 23.6 \\
\hline & $60-69$ & 27 & 0.94 & 0.92 & 0.84 & 5.78 & 0.1 & 58.3 \\
\hline & $70-79$ & 27 & 0.89 & 0.95 & 0.61 & 2.46 & 0.08 & 32.4 \\
\hline & $80-89$ & 20 & 0.97 & 0.92 & 0.83 & 5.35 & 0.1 & 53.2 \\
\hline \multirow[t]{4}{*}{ Female } & $40-59$ & 24.5 & 0.87 & 0.91 & 0.59 & 2.2 & 0.15 & 14.2 \\
\hline & $60-69$ & 16 & 0.91 & 0.9 & 0.83 & 5.32 & 0.12 & 44.2 \\
\hline & $70-79$ & 15 & 0.91 & 0.96 & 0.71 & 3.34 & 0.05 & 64.1 \\
\hline & $80-89$ & 10 & 0.97 & 0.93 & 0.88 & 7.84 & 0.08 & 103.6 \\
\hline
\end{tabular}

Note: If the measured value is equal to or less than the cutoff value, it is determined to be abnormal.

Abbreviations: AUC, area under the curve; LR (+), positive likelihood ratio; LR (-), negative likelihood ratio; DOR, diagnostic odds ratio.

improvement post-DCM surgery. ${ }^{28}$ Grip strength is also frequently assessed in internal medicine to indicate disease activity. ${ }^{14}$ Grip strength has been described as a prognostic indicator of mortality risk in a population of hospitalized older adult women. ${ }^{29}$ Moreover, grip strength has gained attention as an index for evaluating age-related functional decline of motor units such as in sarcopenia. ${ }^{15}$

Screening using a grip strength test is a simple, lowcost, and quantitative method, allowing frequent measurement; thus, satisfying international standards for medical screening. ${ }^{30}$ Inter-rater reliability and test-retest reliability of grip strength have previously been validated. ${ }^{31}$ Furthermore, grip strength can be measured by both physicians and non-medical staff. It may be possible to use grip strength measures not only in primary care but also during routine medical check-ups in the future.

According to previous reports, grip strength is $0.1-$ $10.7 \%$ higher in the dominant hand. ${ }^{32}$ However, in this study, we did not use the influence of the dominant hand for the screening. There are three reasons for this:

1. Using the grip strength of the dominant hand as the cutoff value may miss the onset of the disease when the grip strength of the non-dominant hand decreases.

2. Setting cutoff values for the dominant and nondominant hands is cumbersome and not straightforward.

3. It is difficult to determine ambidextrous patients.

Grip strength can also be decreased due to distal radius fracture, peripheral neuropathy, lateral epicondylitis of the humerus, and arthritis of the carpometacarpal joint. ${ }^{33}$ Thus, it may be difficult to exclude these diseases in primary care. Using the cutoff values established in this study, all patients above the cutoff values would also be referred to a spine specialist for diagnosis and treatment, which is likely to have beneficial results.

\section{Limitations}

This study had several limitations. First, the DCM group comprised surgical patients who were assessed as having severe DCM. However, the diagnostic performance of DCM screening can also be considered valuable in terms of screening to detect DCM that may be severe and requires surgery. Further studies are needed to determine the accuracy of diagnosis in patients with mild DCM. Mild DCM may be missed and must be considered when performing screening. Even when results fall within the normal range, referral to a specialist should be considered for patients with suspected DCM.

Second, a question could be raised concerning the generalizability of the control group results. Participants in the control group were recruited from areas where agriculture is the primary industry. It is possible that these participants may have been more active and had higher muscle strength than those in urban areas. Furthermore, participants in the control group also comprised those who had requested cervical spine examinations during their municipal health checkups. This indicates that this group may have consisted of individuals who were highly conscious of maintaining good health. However, the control group's grip strength in this study was not higher than the national average values reported by the Japan Sports Agency. ${ }^{34}$ In addition, there have been a report that BMI correlates with grip strength, ${ }^{35}$ but these 
relationships were not considered in this study. Given these factors, we consider the control group's results generalizable.

Finally, differences in grip strength in terms of ethnicity should be considered when applying the results of this study. Grip strength values have been reported as being highest among those from Europe/North America, lowest among those from South Asia, South East Asia, and Africa, and intermediate among those from China, South America, and the Middle East. ${ }^{36}$ Cutoff values for grip strength in patients of European ethnicity with sarcopenia have been determined to be $30 \mathrm{~kg}$ for males and $20 \mathrm{~kg}$ for females, whereas among patients of Asian ethnicity, the cutoff values have been determined to be $26 \mathrm{~kg}$ for males and $18 \mathrm{~kg}$ for females. ${ }^{15,20}$ Therefore, it will be necessary to verify the validity of these cutoff values for DCM screening when applied to countries other than Japan in the future.

Despite these limitations, this is the first report to elucidate the diagnostic accuracy of grip strength and relevant cutoff values for DCM screening that could easily be applied in primary care settings. Future studies are needed to validate the usefulness of this screening method for the early detection of DCM.

\section{Conclusion}

We found that grip strength had sufficient diagnostic value for DCM, and we identified cutoff values for screening DCM. This screening method could be useful in primary care for patients that present with a chief complaint of numbness or difficulty using their hands, which is indicative of suspected DCM. This study also suggested that DCM should be differentiated when sarcopenia is identified in primary care.

\section{Acknowledgments}

We would like to thank Editage for English language editing.

\section{Disclosure}

The authors report no conflicts of interest in this work.

\section{References}

1. Nagata K, Yoshimura N, Muraki S, et al. Prevalence of cervical cord compression and its association with physical performance in a population-based cohort in Japan: the Wakayama Spine Study. Spine. 2012;37(22):1892-1898. doi:10.1097/BRS.0b013e31825a2619

2. Nagata K, Yoshimura N, Hashizume $\mathrm{H}$, et al. The prevalence of cervical myelopathy among subjects with narrow cervical spinal canal in a population-based magnetic resonance imaging study: the Wakayama Spine Study. Spine J. 2014;14(12):2811-2817. doi:10.1016/j.spinee.2014.03.051
3. Singh A, Tetreault L, Kalsi-Ryan S, et al. Global prevalence and incidence of traumatic spinal cord injury. Clin Epidemiol. 2014;6:309-331. doi:10.2147/CLEP.S68889

4. Radcliff KE, Curry EP, Trimba R, et al. High incidence of undiagnosed cervical myelopathy in patients with hip fracture compared with controls. J Orthop Trauma. 2016;30(4):189-193. doi:10.1097/ BOT.0000000000000485

5. Kimura A, Seichi A, Takeshita K, et al. Fall-related deterioration of subjective symptoms in patients with cervical myelopathy. Spine. 2017;42(7):E398-E403. doi:10.1097/BRS.0000000000001798

6. Sung RD, Wang JC. Correlation between a positive Hoffmann's reflex and cervical pathology in asymptomatic individuals. Spine. 2001;26(1):67-70. doi:10.1097/00007632-200101010-00013

7. Wong TM, Leung HB, Wong WC. Correlation between magnetic resonance imaging and radiographic measurement of cervical spine in cervical myelopathic patients. $J$ Orthop Surg. 2004;12 (20):239-242. doi:10.1177/230949900401200220

8. Glaser JA, Curé JK, Bailey KL, Morrow DL. Cervical spinal cord compression and the Hoffmann sign. Iowa Orthop J. 2001;21:49-52.

9. Glick TH, Workman TP, Gaufberg SV. Spinal cord emergencies: false reassurance from reflexes. Acad Emerg Med. 1998;5(10):1041-1043. doi:10.1111/j.1553-2712.1998.tb02787.x

10. Crandall PH, Batzdorf U. Cervical spondylotic myelopathy. J Neurosurg. 1966;25(1):57-66. doi:10.3171/jns.1966.25.1.0057

11. Uchihara T, Furukawa T, Tsukagoshi H. Compression of brachial plexus as a diagnostic test of cervical cord lesion. Spine. 1994;19(19):2170-2173. doi:10.1097/00007632-19941000000007

12. Machino M, Imagama S, Ando K, et al. Prospective comparison of age- and sex-related differences in quantifiable $10-\mathrm{S}$ grip and release and 10-S step test results for diagnosis of cervical spondylotic myelopathy in 454 patients with cervical spondylotic myelopathy and 818 asymptomatic subjects. Spine. 2017;42(8):578-585. doi:10.1097/BRS.0000000000001849

13. Kobayashi H, Kikuchi S-I, Otani K, et al. Development of a self-administered questionnaire to screen patients for cervical myelopathy. BMC Musculoskelet Disord. 2010;11(1):268. doi:10.1186/ 1471-2474-11-268

14. Rhind VM, Bird HA, Wright V. A comparison of clinical assessments of disease activity in rheumatoid arthritis. Ann Rheum Dis. 1980;39 (2):135-137. doi:10.1136/ard.39.2.135

15. Cruz-Jentoft AJ, Baeyens JP, Bauer JM, et al. Sarcopenia: European consensus on definition and diagnosis: report of the European working group on sarcopenia in older people. Age Ageing. 2010;39 (4):412-423. doi:10.1093/ageing/afq034

16. Otani K, Kikuchi SI, Yabuki S, et al. Prospective one-year follow-up of lumbar spinal stenosis in a regional community. $J$ Pain Res. 2018;2(11):455-464. doi:10.2147/JPR.S148402

17. Jackson R. The Cervical Syndrome. Thomas CC, editor. Oxford: Blackwell Scientific Publications; 1966.

18. Spurling RS, Scoville WB. Lateral rupture of the cervical intervertebral discs: a common cause of shoulder and arm pain. Surg Gynecol Obstet. 1944;78:350-358.

19. Fischer JE, Bachmann LM, Jaeschke R. A readers' guide to the interpretation of diagnostic test properties: clinical example of sepsis. Intensive Care Med. 2003;29(7):1043-1051. doi:10.1007/s00134003-1761-8

20. Chen LK, Woo J, Assantachai P, et al. Asian Working Group for Sarcopenia: 2019 consensus update on sarcopenia diagnosis and treatment. $J$ Am Med Dir Assoc. 2020;21(3):300-307.e2. doi:10.1016/j.jamda.2019.12.012

21. Machino M, Ando K, Kobayashi K, et al. Cut off value in each gender and decade of 10-s grip and release and 10-s step test: a comparative study between 454 patients with cervical spondylotic myelopathy and 818 healthy subjects. Clin Neurol Neurosurg. 2019;184:105414. doi:10.1016/j.clineuro.2019.105414 
22. Fukui M, Chiba K, Kawakami M, et al. Japanese Orthopaedic Association Cervical Myelopathy Evaluation Questionnaire (JOACMEQ): part 2. Endorsement of the alternative item. J Orthop Sci. 2007;12(3):241-248. doi:10.1007/s00776-007-1119-0

23. Fukui M, Chiba K, Kawakami M, et al. Japanese Orthopaedic Association Cervical Myelopathy Evaluation Questionnaire: part 3. Determination of reliability. J Orthop Sci. 2007;12(4):321-326. doi:10.1007/s00776-007-1131-4

24. Fukui M, Chiba K, Kawakami M, et al. An outcome measure for patients with cervical myelopathy: Japanese Orthopaedic Association Cervical Myelopathy Evaluation Questionnaire (JOACMEQ): part 1. J Orthop Sci. 2007;12(3):227-240. doi:10.1007/s00776-007-1118-1

25. Fukui M, Chiba K, Kawakami M, et al. Japanese Orthopaedic Association Cervical Myelopathy Evaluation Questionnaire (JOACMEQ): part 4. Establishment of equations for severity scores. Subcommittee on low back pain and cervical myelopathy, evaluation of the clinical outcome committee of the Japanese Orthopaedic Association. J Orthop Sci. 2008;13(1):25-31. doi:10.1007/s00776-007-1194-2

26. Bishop PB, Wing PC. Knowledge transfer in family physicians managing patients with acute low back pain: a prospective randomized control trial. Spine J. 2006;6(3):282-288. doi:10.1016/j. spinee.2005.10.008

27. Rice CL, Cunningham DA, Paterson DH, et al. Strength in an elderly population. Arch Phys Med Rehabil. 1989;70:391-397.

28. Suzuki A, Misawa H, Simogata M, et al. Recovery process following cervical laminoplasty in patients with cervical compression myelopathy: prospective cohort study. Spine. 2009;34(26):2874-2879. doi:10.1097/BRS.0b013e3181bb0e33
29. Phillips P. Grip strength, mental performance and nutritional status as indicators of mortality risk among female geriatric patients. Age Ageing. 1986;15(1):53-56. doi:10.1093/ageing/15.1.53

30. Wilson JMG, Jungner G. Principles and practice of screening for disease. WHO papers No 34. WHO; 1969. Available from: https:// apps.who.int/iris/handle/10665/37650. Accessed November 18, 2021.

31. Mathiowetz V, Weber K, Volland G, et al. Reliability and validity of grip and pinch strength evaluations. J Hand Surg Am. 1984;9 (2):222-226. doi:10.1016/S0363-5023(84)80146-X

32. Bohannon RW. Grip strength: a summary of studies comparing dominant and nondominant limb measurements. Percept Mot Skills. 2003;96(3):728-730. doi:10.2466/pms.2003.96.3.728

33. Bobos P, Nazari G, Lu Z, et al. Measurement properties of the hand grip strength assessment: a systematic review with meta-analysis. Arch Phys Med Rehabil. 2020;101(3):553-565. doi:10.1016/j. apmr.2019.10.183

34. Japan Sports Agency. Overview of the 2008 physical fitness and athletic performance survey. Available from: https:/www.mext.go. jp/prev_sports/comp/b_menu/other/_icsFiles/afieldfile/2019/10/15/ 1421921_1.pdf. Accessed January 31, 2021.

35. Liao KH. Hand grip strength in low, medium, and high body mass index males and females. Middle East J Rehabil Health. 2016;3(1). doi:10.17795/mejrh-33860

36. Leong DP, Teo KK, Rangarajan S, et al. Reference ranges of handgrip strength from 125,462 healthy adults in 21 countries: a prospective urban rural epidemiologic (PURE) study. J Cachexia Sarcopenia Muscle. 2016;7(5):535-546. doi:10.1002/ jesm. 12112
International Journal of General Medicine

\section{Publish your work in this journal}

The International Journal of General Medicine is an international, peer-reviewed open-access journal that focuses on general and internal medicine, pathogenesis, epidemiology, diagnosis, monitoring and treatment protocols. The journal is characterized by the rapid reporting of reviews, original research and clinical studies

\section{Dovepress}

across all disease areas. The manuscript management system is completely online and includes a very quick and fair peer-review system, which is all easy to use. Visit http://www.dovepress.com testimonials.php to read real quotes from published authors. 